\title{
CONSENSUS CONTROL FOR LEADER-FOLLOWING MULTI-AGENT SYSTEMS WITH MEASUREMENT NOISES*
}

\author{
Cuiqin MA · Tao LI · Jifeng ZHANG
}

DOI: $10.1007 / \mathrm{s} 11424-010-9273-4$

Received: 27 May 2009

(C)The Editorial Office of JSSC \& Springer-Verlag Berlin Heidelberg 2010

\begin{abstract}
This work is concerned with consensus control for a class of leader-following multi-agent systems (MASs). The information that each agent received is corrupted by measurement noises. To reduce the impact of noises on consensus, time-varying consensus gains are adopted, based on which consensus protocols are designed. By using the tools of stochastic analysis and algebraic graph theory, a sufficient condition is obtained for the protocol to ensure strong mean square consensus under the fixed topologies. This condition is shown to be necessary and sufficient in the noise-free case. Furthermore, by using a common Lyapunov function, the result is extended to the switching topology case.
\end{abstract}

Key words Consensus protocol, leader-following, multi-agent system, stochastic noise.

\section{Introduction}

The leader-following MAS is an important kind of MASs. It has been extensively used to represent systems in many practical applications such as biological systems ${ }^{[1]}$, formation control $^{[2]}$ and large scale robotic systems ${ }^{[3]}$, etc. In leader-following MASs, the leaders are usually independent of their followers, but have influence on the followers' behaviors. Therefore, one can realize one's control objective of the agents by only controlling the leaders, which transfers the control of the whole system to that of a single-agent. This not only simplifies the design and implementation of the controls but also helps to save energy and reduce control $\operatorname{costs}^{[4-5]}$.

Consensus control for leader-following MASs has attracted much attention. For instance, Jadbabaie et al. ${ }^{[6]}$ considered the nearest neighborhood principle, and under time-varying topologies, proved that if all the agents were jointly connected with their leader, then their states would converge to the state of the leader as time goes on. Ren and Beard ${ }^{[7]}$ extended the results of $[6]$ to the directed topology case. Hong et al. ${ }^{\left[{ }^{[}\right]}$considered the multi-agent consensus with an active leader and variable interconnection topology.

A common feature of the existing works on consensus control for leader-following MASs is that measurement noises are not considered and the state information is assumed to be

Cuiqin MA

School of Mathematical Sciences, Qufu Normal University, Qufu 273165, China. Email: cuiqinma@amss.ac.cn. Tao LI · Jifeng ZHANG

Key Laboratory of Systems and Control, Institute of Systems Science, Academy of Mathematics and Systems Science, Chinese Academy of Sciences, Beijing 100190, China. Email: litao@amss.ac.cn; jif@iss.ac.cn.

* This research was supported by the National Natural Science Foundation of China under Grant Nos. 60821091 and 60934006. Part of this work was presented at the 17th IFAC World Congress, Seoul, Korea, July 2008. 
exactly obtained. However, real communication processes are often disturbed by various random factors. Therefore, just as Ren et al. ${ }^{[9]}$ pointed out, for the consensus problems it is important to investigate how to design consensus protocols applicable to the cases with communication noises. This paper will focus on leader-following consensus control under measurement noises.

For leader-following MASs, the main difficulties of consensus control under measurement noises stem from noise disturbance and state coupling of the closed-loop system. Here, measurement noises come into the consensus protocol, which renders the closed-loop system a stochastic differential equation. Also, the communication topology among agents in the MAS is described by a digraph. This makes the state matrix of the above stochastic differential equation neither symmetric nor diagonalizable since the Laplacian matrix of a digraph is nonsymmetric. Thus, the closed-loop system cannot be decoupled, and hence, it is hard to analyze the convergence of the consensus protocol.

It is worth pointing out that different from [10] and [11], here the digraph is not assumed to be balanced, and thus, the method of the symmetrized graph in [10] is not suitable to the convergence analysis. Also, unlike the noise-free cases (e.g. [6, 12]) where the convergence properties of the protocol are often analyzed by employing the theory of stochastic matrices, here due to the existence of measurement noises, the state matrix of the closed-loop equation is no longer a stochastic matrix, and thus, the tools of stochastic matrices do not work.

With these observations, to overcome the impact of measurement noises, time-varying consensus gains are introduced into the consensus protocol designed. And convergence properties of the protocol are analyzed by using a common Lyapunov function. Under fixed topologies, a sufficient condition is obtained for the state of each follower to converge to that of the leader in mean square, which is also found necessary and sufficient in the noise-free case. In addition, under switching topologies, the protocol is also shown to be a strong mean square consensus protocol if the subgraph formed by the followers is balanced.

The remainder of this paper is organized as follows. Some preliminary results of graph theory are briefly reviewed in Section 2. The problem to be investigated is formulated in Section 3. The consensus protocols are designed in Section 4, and in Section 5 the convergence properties of the closed-loop system are analyzed under fixed and switching topologies, respectively. A numerical example is given in Section 6 to illustrate our results. Finally, some concluding remarks and future research topics are discussed in Section 7.

The following notations will be used throughout this paper: $\mathbb{R}^{m \times n}$ denotes the family of $m \times n$ dimensional real matrices; $I_{m}$ denotes the $m \times m$ dimensional identity matrix; 1 denotes a column vector with all ones; $\mathbb{R}$ denotes the real number field; $\lambda_{\max }(X)$ and $\lambda_{\min }(X)$ denote the maximum and minimum eigenvalues of the real symmetric matrix $X$, respectively. For a given vector or matrix $X, X^{\mathrm{T}}$ denotes its transpose; $\operatorname{tr}(X)$ denotes its trace; $\|X\|$ denotes its Euclidean norm. For a given set $S, \chi_{S}$ denotes the indicator function of $S$. For a family of random variables $\left\{\xi_{\lambda}, \lambda \in \Lambda\right\}, \sigma\left(\xi_{\lambda}, \lambda \in \Lambda\right)$ denotes the $\sigma$-algebra generated by $\left\{\xi_{\lambda} \in B, B \in \mathcal{B}, \lambda \in \Lambda\right\}$ where $\mathcal{B}$ denotes the 1 -dimensional Borel sets.

\section{Preliminaries}

In this paper, we model information exchange among agents in MASs by digraphs. Thus, before formulating our problem, we first introduce some basic notations from digraphs ${ }^{[4,9,12]}$.

Let $\mathcal{G}=(\mathcal{V}, \mathcal{E})$ be a digraph with the set of vertices $\mathcal{V}=\{1,2, \cdots, N\}$ and the set of edges $\mathcal{E} \subseteq \mathcal{V} \times \mathcal{V}$. In $\mathcal{G}$, the $i$ th vertex represents the $i$ th agent, and a directed edge from $i$ to $j$ is denoted as an ordered pair $(i, j) \in \mathcal{E}$, which means that agent $j$ can directly receive information from agent $i$. If there is a directed edge from $i$ to $j$, then the vertex $i$ is called the parent vertex 
and the vertex $j$ is called the child vertex. The set of neighbors of the $i$ th agent is denoted by $N_{i}=\{j \in \mathcal{V} \mid(j, i) \in \mathcal{E}\}$. $(i, i)$ is called a self-loop. In this paper, we assume that the digraph has no self-loops, unless stated otherwise.

A sequence of edges $\left(i_{1}, i_{2}\right),\left(i_{2}, i_{3}\right), \cdots,\left(i_{k-1}, i_{k}\right)$ is called a directed path from vertex $i_{1}$ to vertex $i_{k}$. A digraph is called strongly connected if there is a directed path from every node to every other node. A directed tree is such a digraph whose every vertex except the root, which has only children but no parent, has exactly one parent. A spanning tree of a digraph is a directed tree that contains all the vertices of the digraph.

Suppose $\mathcal{G}=(\mathcal{V}, \mathcal{E})$ and $\overline{\mathcal{G}}=(\overline{\mathcal{V}}, \overline{\mathcal{E}})$ are two digraphs. If $\overline{\mathcal{V}} \subseteq \mathcal{V}$ and $\overline{\mathcal{E}} \subseteq \mathcal{E}$, then $\overline{\mathcal{G}}$ is said to be a subgraph of $\mathcal{G}$.

$\mathcal{A}=\left(a_{i j}\right) \in \mathbb{R}^{N \times N}$ is called the weighted adjacency matrix of $\mathcal{G}=(\mathcal{V}, \mathcal{E})$ with nonnegative elements, and $a_{i i}=0, a_{i j}>0 \Leftrightarrow j \in N_{i}$. Specially, if $a_{i j}=a_{j i}, i, j=1,2, \cdots, N$, then $\mathcal{G}=(\mathcal{V}, \mathcal{E}, \mathcal{A})$ is said to be an undirected graph. The in-degree and out-degree of vertex $i$ are defined as $\operatorname{deg}_{i n}(i)=\sum_{j=1}^{N} a_{i j}$ and $\operatorname{deg}_{\text {out }}(i)=\sum_{j=1}^{N} a_{j i}$, respectively. If $\operatorname{deg}_{i n}(i)=$ $\operatorname{deg}_{\text {out }}(i), i=1,2, \cdots, N$, then $\mathcal{G}$ is called balanced.

The Laplacian matrix of the weighted digraph $\mathcal{G}=(\mathcal{V}, \mathcal{E}, \mathcal{A})$ is defined as $L_{\mathcal{G}}=\mathcal{D}-\mathcal{A}$, where $\mathcal{D}=\operatorname{diag}\left(\operatorname{deg}_{i n}(1), \operatorname{deg}_{i n}(2), \cdots, \operatorname{deg}_{i n}(N)\right)$.

Let $\mathcal{G}=(\mathcal{V}, \mathcal{E}, \mathcal{A})$ be a weighted digraph and $\widetilde{\mathcal{E}}=\{(j, i) \mid(i, j) \in \mathcal{E}\}$. The mirror of $\mathcal{G}$ denoted by $\widehat{\mathcal{G}}=(\mathcal{V}, \widehat{\mathcal{E}}, \widehat{\mathcal{A}})$ is an undirected graph, where the set of edges $\widehat{\mathcal{E}}=\mathcal{E} \cup \widetilde{\mathcal{E}}$, and the symmetric weighted adjacency matrix $\widehat{\mathcal{A}}=\left(\widehat{a}_{i j}\right)$ with elements $\widehat{a}_{i j}=\widehat{a}_{j i}=\frac{a_{i j}+a_{j i}}{2} \geq 0$.

Below are important results for the Laplacian matrix.

Lemma $1^{[13]} \quad$ Suppose that $\mathcal{G}=(\mathcal{V}, \mathcal{E}, \mathcal{A})$ is a weighted digraph and $\widehat{\mathcal{G}}=(\mathcal{V}, \widehat{\mathcal{E}}, \widehat{\mathcal{A}})$ is the mirror digraph of $\mathcal{G}$. Then, $\frac{L_{\mathcal{G}}+L_{\mathcal{G}}^{\mathrm{T}}}{2}$ is the Laplacian matrix of $\widehat{\mathcal{G}}$ if and only if $\mathcal{G}$ is balanced, where $L_{\mathcal{G}}$ is the Laplacian matrix of $\mathcal{G}$.

Lemma $2^{[4]}$ The Laplacian matrix $L_{\mathcal{G}}$ of a digraph $\mathcal{G}=(\mathcal{V}, \mathcal{E}, \mathcal{A})$ has at least one zero eigenvalue and all the nonzero eigenvalues are in the open right half plane. Furthermore, $L_{\mathcal{G}}$ has exactly one zero eigenvalue if and only if $\mathcal{G}$ has a spanning tree.

\section{Problem Formulation}

Here, we consider a system consisting of $N+1$ agents where an agent indexed by 0 acts as the leader and the other agents indexed by $1,2, \cdots, N$, respectively, act as the followers. The dynamics of the $i$ th follower is described as follows:

$$
\dot{x}_{i}(t)=u_{i}(t), \quad i=1,2, \cdots, N,
$$

where $x_{i}(t) \in \mathbb{R}$ and $u_{i}(t) \in \mathbb{R}$ are the state and control input of the $i$ th follower, respectively. In general, the behavior of the leader is independent of the followers. $x_{0}$ denotes the state of the leader and keeps being a constant.

With regarding the $N+1$ agents as vertices, the topology relationships among them can be conveniently described by a $\operatorname{digraph} \mathcal{G}=(\mathcal{V}, \mathcal{E}, \mathcal{A})$ with

$$
\mathcal{V}=\{0,1,2, \cdots, N\}, \quad \mathcal{A}=\left(\begin{array}{cccc}
0 & 0 & \cdots & 0 \\
a_{10} & 0 & \cdots & a_{1 N} \\
\vdots & \vdots & \ddots & \vdots \\
a_{N 0} & a_{N 1} & \cdots & 0
\end{array}\right) \in \mathbb{R}^{(N+1) \times(N+1)}
$$


For simplicity, let $B=\operatorname{diag}\left(b_{1}, b_{2}, \cdots, b_{N}\right)$ represent the leader adjacency matrix associated with $\mathcal{G}$, where $b_{i}=a_{i 0} \geq 0$. Obviously, $b_{i}>0 \Leftrightarrow 0 \in N_{i}$. Let the $\operatorname{digraph} \overline{\mathcal{G}}=(\overline{\mathcal{V}}, \overline{\mathcal{E}}, \overline{\mathcal{A}})$ represent the subgraph formed by the $N$ followers, where

$$
\overline{\mathcal{V}}=\mathcal{V} \backslash\{0\}, \quad \overline{\mathcal{A}}=\left(\begin{array}{ccc}
0 & \cdots & a_{1 N} \\
\vdots & \ddots & \vdots \\
a_{N 1} & \cdots & 0
\end{array}\right) \in \mathbb{R}^{N \times N}
$$

As it is well-known, real communication processes are often corrupted by random noises. In our models, the $i$ th agent receives information from its neighbors with measurement noises:

$$
y_{j i}(t)=x_{j}(t)+n_{j i}(t), \quad j \in N_{i}
$$

where $y_{j i}(t)$ denotes the measurement of the $j$ th agent's state $x_{j}(t)$ by the $i$ th agent, and $\left\{n_{j i}(t), j \in N_{i}, i=1,2, \cdots, N\right\}$ are independent standard white noises.

A group of controls $\mathcal{U}=\left\{u_{i}, i=1,2, \cdots, N\right\}$ is called a measurement-based distributed protocol $^{[10]}$, if

$$
u_{i}(t) \in \sigma\left(x_{i}(s), \bigcup_{j \in N_{i}} y_{j i}(s), 0 \leq s \leq t\right), \forall t \geq 0, i=1,2, \cdots, N .
$$

The so-called consensus problem for leader-following MASs under measurement noises is to design a measurement-based distributed protocol such that as time goes on, each follower's state will finally converge to the leader's.

This paper is to investigate the above problem. First, we design the consensus protocol for each follower.

\section{Consensus Protocol}

We propose a protocol for the $i$ th follower as

$$
u_{i}(t)=a(t)\left[\sum_{j \in N_{i}} a_{i j}\left(y_{j i}(t)-x_{i}(t)\right)+b_{i}\left(y_{0 i}(t)-x_{i}(t)\right)\right], t \geq 0, i=1,2, \cdots, N,
$$

where $a(\cdot):[0, \infty) \longrightarrow(0, \infty)$ is a piecewise continuous function, usually called a time-varying consensus gain ${ }^{[10]}$ and $a_{i j}$ is defined as in $(2) ; N_{i}=N_{i}(\mathcal{G})$ denotes the neighbor set of agent $i$ and varies with $\mathcal{G}$ in the switching topology case.

Remark 1 i) Different from the leader-following consensus protocols in [6-8], the measurement noises are explicitly taken into account in the consensus protocol (3).

ii) From (3) it is obvious that the consensus protocol devised for the $i$ th follower is indeed a measurement-based distributed protocol since it depends only on the state information of itself and its neighbors'.

Next, a natural question is that after applying (3), whether the state of the $i$ th follower will converge to that of the leader? To answer this question, we first investigate the closed-loop system based on (3).

Let $\alpha_{i}$ represent the $i$ th row of the matrix $\overline{\mathcal{A}}, H=\operatorname{diag}\left(\alpha_{1}, \alpha_{2}, \cdots, \alpha_{N}\right)$ which is an $N \times N^{2}$ dimensional block diagonal matrix, $n_{0}(t)=\left(n_{01}, n_{02}, \cdots, n_{0 N}\right)^{\mathrm{T}}, n_{i}(t)=\left(n_{1 i}, n_{2 i}, \cdots, n_{N i}\right)^{\mathrm{T}}$, $i=1,2, \cdots, N$, and $Q=(B, H)$ which is an $N \times\left(N^{2}+N\right)$ dimensional block matrix. 
Denote $X(t)=\left(x_{1}(t), x_{2}(t), \cdots, x_{N}(t)\right)^{\mathrm{T}}$. Substituting the consensus protocol (3) to the system (1), we have

$$
\frac{d X(t)}{d t}=a(t)\left(-L_{\overline{\mathcal{G}}}-B\right) X(t)+a(t) B\left(\mathbf{1} \otimes x_{0}\right)+a(t) Q Z(t),
$$

where $\otimes$ denotes the Kronecker product, $L_{\overline{\mathcal{G}}}$ is the Laplacian matrix of $\overline{\mathcal{G}}$ and $Z(t)=\left(n_{0}^{\mathrm{T}}(t), n_{1}^{\mathrm{T}}(t)\right.$, $\left.\cdots, n_{N}^{\mathrm{T}}(t)\right)^{\mathrm{T}}$ is an $N^{2}+N$ dimensional independent standard white noise sequence.

We can construct an $N^{2}+N$ dimensional standard Brownian motion $W^{*}(t)=\left(W_{01}(t), \cdots\right.$, $\left.W_{0 N}(t), W_{11}(t), \cdots, W_{N 1}(t), \cdots, W_{1 N}(t), \cdots, W_{N N}(t)\right)^{\mathrm{T}}$, and rewrite $(4)$ as

$$
d X(t)=a(t)\left(-L_{\overline{\mathcal{G}}}-B\right) X(t) d t+a(t) B\left(\mathbf{1} \otimes x_{0}\right) d t+a(t) Q d W^{*}(t) .
$$

To facilitate analysis, it is convenient to construct an $N$ dimensional standard Brownian motion $W(t)=\left(W_{1}(t), W_{2}(t), \cdots, W_{N}(t)\right)^{\mathrm{T}}$ where

$$
W_{i}(t)=\left\{\begin{array}{cc}
\frac{b_{i} W_{0 i}(t)+\sum_{j \in N_{i}} a_{i j} W_{j i}(t)}{\sqrt{\bar{b}_{i}^{2}+\sum_{j \in N_{i}} a_{i j}^{2}}}, & b_{i}^{2}+\sum_{j \in N_{i}} a_{i j}^{2} \neq 0 ; \\
W_{0 i}(t), & b_{i}^{2}+\sum_{j \in N_{i}} a_{i j}^{2}=0 .
\end{array}\right.
$$

Combining with (5), we get

$$
d X(t)=a(t)\left(-L_{\overline{\mathcal{G}}}-B\right) X(t) d t+a(t) B\left(\mathbf{1} \otimes x_{0}\right) d t+a(t) G d W(t),
$$

where

$$
G=\operatorname{diag}\left(\sqrt{b_{1}^{2}+\sum_{j \in N_{1}} a_{1 j}^{2}}, \sqrt{b_{2}^{2}+\sum_{j \in N_{2}} a_{2 j}^{2}}, \cdots, \sqrt{b_{N}^{2}+\sum_{j \in N_{N}} a_{N j}^{2}}\right) .
$$

In the sequel, we will show that under the consensus protocol (3), each follower's state will converge to the leader's.

\section{Convergence Analysis}

After applying the consensus protocol (3), the closed-loop system (6) is stochastic, since (3) takes into account measurement noises. Thus, before establishing the convergence properties of the consensus protocol (3), we first introduce a definition of consensus protocols for stochastic systems.

Definition $1^{[14]}$ A distributed protocol $\mathcal{U}=\left\{u_{i}, i=1,2, \cdots, N\right\}$ is called a strong mean square consensus protocol if $\mathcal{U}=\left\{u_{i}, i=1,2, \cdots, N\right\}$ renders the system (1) has the following properties:

$$
\lim _{t \rightarrow \infty} E\left(x_{i}(t)-x^{*}\right)^{2}=0, \quad i=1,2, \cdots, N,
$$

where $x^{*}$ is a random variable and $E\left(x^{*}\right)^{2}<\infty$.

For the system (1), we make the following assumptions:

$\left(\mathbf{A}_{\mathbf{1}}\right) \mathcal{G}=(\mathcal{V}, \mathcal{E}, \mathcal{A})$ has a spanning tree.

$\left(\mathbf{A}_{\mathbf{2}}\right) \quad \int_{0}^{\infty} a(s) d s=\infty$.

(A $\left.\mathbf{A}_{3}\right) \int_{0}^{\infty} a^{2}(s) d s<\infty$. 
Remark 2 i) Generally speaking, a digraph does not always have a spanning tree. However, a strongly connected digraph must have a spanning tree. Therefore, Assumption $\left(\mathbf{A}_{\mathbf{1}}\right)$ is weaker than the strong connectivity condition.

ii) $\left(\mathbf{A}_{\mathbf{2}}\right)-\left(\mathbf{A}_{\mathbf{3}}\right)$ are standard assumptions often used in stochastic approximation ${ }^{[15]}$, which (especially $\left(\mathbf{A}_{\mathbf{2}}\right)$ ) in some cases happen to be the weakest conditions to ensure a consensus protocol (see Theorem 2 below).

Denote $\delta(t)=X(t)-\mathbf{1} \otimes x_{0}$. Then, by (6) we have

$$
d \delta(t)=a(t)\left(-L_{\overline{\mathcal{G}}}-B\right) \delta(t) d t+a(t) G d W(t) .
$$

Next, we will demonstrate that the consensus protocol (3) is a strong mean square consensus protocol under Assumptions $\left(\mathbf{A}_{\mathbf{1}}\right)-\left(\mathbf{A}_{\mathbf{3}}\right)$.

\subsection{Fixed Topology}

Theorem 1 For the system (1) with the protocol (3), if Assumptions $\left(\mathbf{A}_{\mathbf{1}}\right)-\left(\mathbf{A}_{\mathbf{3}}\right)$ hold, then

$$
\lim _{t \rightarrow \infty} E\|\delta(t)\|^{2}=0
$$

that is, (3) is a strong mean square consensus protocol.

Proof Noticing the definition of the matrix $B$ and the fact that $L_{\overline{\mathcal{G}}}$ is the Laplacian matrix of $\overline{\mathcal{G}}$, we know that

$$
L_{\mathcal{G}}=\left(\begin{array}{cc}
0 & 0 \\
-B \cdot \mathbf{1} & L_{\overline{\mathcal{G}}}+B
\end{array}\right)
$$

is the Laplacian matrix of $\mathcal{G}$. Consequently, from Assumption $\left(\mathbf{A}_{\mathbf{1}}\right)$ and Lemma 2, it follows that $L_{\mathcal{G}}$ has exactly one zero eigenvalue and all of the nonzero eigenvalues are in the open right half plane. Thus, all the eigenvalues of $L_{\overline{\mathcal{G}}}+B$ are in the open right half plane, and hence, $-L_{\overline{\mathcal{G}}}-B$ is a stable matrix, and the Lyapunov equation

$$
\left(-L_{\overline{\mathcal{G}}}-B\right) P+P\left(-L_{\overline{\mathcal{G}}}-B\right)^{\mathrm{T}}=-I_{N}
$$

has a unique positive definite solution $P$. Let

$$
V(t)=\delta^{\mathrm{T}}(t) P \delta(t),
$$

Then, by (7) and the Itô formula, we get

$$
d V(t)=-a(t) \delta^{\mathrm{T}}(t) \delta(t) d t+a^{2}(t) \operatorname{tr}\left(P G G^{\mathrm{T}}\right) d t+2 a(t) \delta^{\mathrm{T}}(t) P G d W(t) .
$$

Noticing that $P>0$, we have

$$
d V(t) \leq-\frac{a(t)}{\lambda_{\max }(P)} V(t) d t+a^{2}(t) \operatorname{tr}\left(P G G^{\mathrm{T}}\right) d t+2 a(t) \delta^{\mathrm{T}}(t) P G d W(t) .
$$

Now, we prove that

$$
E \int_{t_{0}}^{t} a(s) \delta^{\mathrm{T}}(s) P G d W(s)=0, \quad \forall 0 \leq t_{0} \leq t .
$$


For any given $t_{0} \geq 0, T \geq t_{0}$, let $\tau_{K}^{t_{0}}=\inf \left\{t \geq t_{0} \mid \delta^{\mathrm{T}}(t) P \delta(t) \geq K\right\}$, where $K$ is a given positive integer. From (10) one can get

$$
\begin{aligned}
& E\left[V\left(t \wedge \tau_{K}^{t_{0}}\right) \chi_{\left\{t \leq \tau_{K}^{t_{0}}\right\}}\right]-E\left[V\left(t_{0}\right)\right] \\
\leq & -\frac{1}{\lambda_{\max }(P)} \int_{t_{0}}^{t} a(s) E\left[V\left(s \wedge \tau_{K}^{t_{0}}\right) \chi_{\left\{s \leq \tau_{K}^{t_{0}}\right\}}\right] d s+\operatorname{tr}\left(P G G^{\mathrm{T}}\right) \int_{t_{0}}^{t} a^{2}(s) d s \\
\leq & \operatorname{tr}\left(P G G^{\mathrm{T}}\right) \int_{t_{0}}^{\mathrm{T}} a^{2}(s) d s, \quad \forall t_{0} \leq t \leq T,
\end{aligned}
$$

which implies that there exists a constant $M_{t_{0}, T}>0$ such that

$$
E\left[V\left(t \wedge \tau_{K}^{t_{0}}\right) \chi_{\left\{t \leq \tau_{K}^{t_{0}}\right\}}\right] \leq M_{t_{0}, T}<\infty, \quad \forall 0 \leq t_{0} \leq T .
$$

Noticing that $t \wedge \tau_{K}^{t_{0}} \underset{K \rightarrow \infty}{\stackrel{\text { a.s. }}{\longrightarrow}} t$, by the above inequality and the Fatou lemma, we have

$$
\sup _{t_{0} \leq t \leq T} E[V(t)] \leq M_{t_{0}, T}
$$

Thus,

$$
E \int_{t_{0}}^{t} a^{2}(s) V(s) d s \leq \sup _{t_{0} \leq t \leq T} E[V(t)] \int_{t_{0}}^{T} a^{2}(s) d s<\infty, \quad \forall t_{0} \leq t \leq T .
$$

By the arbitrariness of $T$, we obtain

$$
E \int_{t_{0}}^{t} a^{2}(s) V(s) d s<\infty, \quad \forall 0 \leq t_{0} \leq t .
$$

This together with

$$
E \int_{t_{0}}^{t} a^{2}(s)\left\|\delta^{\mathrm{T}}(s) P G\right\|^{2} d s \leq\|P\|\|G\|^{2} E \int_{t_{0}}^{t} a^{2}(s) V(s) d s
$$

gives (11). From (10) it follows that for any $t \geq 0$ and $h>0$,

$$
E[V(t+h)]-E[V(t)] \leq-\frac{1}{\lambda_{\max }(P)} \int_{t}^{t+h} a(s) E[V(s)] d s+\operatorname{tr}\left(P G G^{\mathrm{T}}\right) \int_{t}^{t+h} a^{2}(s) d s,
$$

or equivalently,

$$
\frac{E[V(t+h)]-E[V(t)]}{h} \leq \frac{-\frac{1}{\lambda_{\max }(P)} \int_{t}^{t+h} a(s) E[V(s)] d s+\operatorname{tr}\left(P G G^{\mathrm{T}}\right) \int_{t}^{t+h} a^{2}(s) d s}{h} .
$$

Further,

$$
\begin{aligned}
\limsup _{h \rightarrow 0^{+}} \frac{E[V(t+h)]-E[V(t)]}{h} & \leq \limsup _{h \rightarrow 0^{+}} \frac{-\frac{1}{\lambda_{\max }(P)} \int_{t}^{t+h} a(s) E[V(s)] d s+\operatorname{tr}\left(P G G^{\mathrm{T}}\right) \int_{t}^{t+h} a^{2}(s) d s}{h} \\
& =-\frac{1}{\lambda_{\max }(P)} a(t) E[V(t)]+\operatorname{tr}\left(P G G^{\mathrm{T}}\right) a^{2}(t) .
\end{aligned}
$$


Thus, by the comparison principle ${ }^{[16]}$, we have that for any $t \in[0, t+h]$,

$$
\begin{aligned}
E[V(t)] \leq & E[V(0)] \exp \left\{-\frac{1}{\lambda_{\max }(P)} \int_{0}^{t} a(s) d s\right\} \\
& +\operatorname{tr}\left(P G G^{\mathrm{T}}\right) \int_{0}^{t} a^{2}(s) \exp \left\{-\frac{1}{\lambda_{\max }(P)} \int_{s}^{t} a(\tau) d \tau\right\} d s .
\end{aligned}
$$

By Assumption $\left(\mathbf{A}_{\mathbf{3}}\right)$, for any given $\varepsilon>0$, there exists $s_{0}>0$ such that $\int_{s_{0}}^{\infty} a^{2}(s) d s<\varepsilon$. Hence,

$$
\begin{aligned}
& \operatorname{tr}\left(P G G^{\mathrm{T}}\right) \int_{0}^{t} a^{2}(s) \exp \left\{-\frac{1}{\lambda_{\max }(P)} \int_{s}^{t} a(\tau) d \tau\right\} d s \\
= & \operatorname{tr}\left(P G G^{\mathrm{T}}\right) \int_{0}^{s_{0}} a^{2}(s) \exp \left\{-\frac{1}{\lambda_{\max }(P)} \int_{s}^{t} a(\tau) d \tau\right\} d s \\
& +\operatorname{tr}\left(P G G^{\mathrm{T}}\right) \int_{s_{0}}^{t} a^{2}(s) \exp \left\{-\frac{1}{\lambda_{\max }(P)} \int_{s}^{t} a(\tau) d \tau\right\} d s \\
\leq & \operatorname{tr}\left(P G G^{\mathrm{T}}\right) \exp \left\{-\frac{1}{\lambda_{\max }(P)} \int_{s_{0}}^{t} a(\tau) d \tau\right\} \int_{0}^{s_{0}} a^{2}(s) d s+\operatorname{tr}\left(P G G^{\mathrm{T}}\right) \int_{s_{0}}^{t} a^{2}(s) d s \\
\leq & \operatorname{tr}\left(P G G^{\mathrm{T}}\right) \exp \left\{-\frac{1}{\lambda_{\max }(P)} \int_{s_{0}}^{t} a(\tau) d \tau\right\} \int_{0}^{\infty} a^{2}(s) d s+\operatorname{tr}\left(P G G^{\mathrm{T}}\right) \int_{s_{0}}^{\infty} a^{2}(s) d s \\
\leq & o(1)+\operatorname{tr}\left(P G G^{\mathrm{T}}\right) \varepsilon, \quad t \rightarrow \infty .
\end{aligned}
$$

Since $\varepsilon$ is arbitrary,

$$
\lim _{t \rightarrow \infty} \operatorname{tr}\left(P G G^{\mathrm{T}}\right) \int_{0}^{t} a^{2}(s) \exp \left\{-\frac{1}{\lambda_{\max }(P)} \int_{s}^{t} a(\tau) d \tau\right\} d s=0 .
$$

Noticing that $\|\delta(t)\|^{2} \leq \frac{V(t)}{\lambda_{\min }(P)}$, by Assumption $\left(\mathbf{A}_{\mathbf{2}}\right)$ and $(12)$, (8) holds.

Remark 3 From Theorem 1 one can see that for the fixed topology case, under Assumptions $\left(\mathbf{A}_{\mathbf{1}}\right)-\left(\mathbf{A}_{\mathbf{3}}\right)$, the designed protocol ensures that the state of each follower converges to that of the leader in mean square.

Remark 4 Different from [10] and [11], here we only require $\mathcal{G}$ has a spanning tree, and do not require it is balanced.

It is worth pointing out that, unlike [6] and [12], here random measurement noises are considered. To reduce the influence of noises, time-varying consensus gains are adopted, which renders the closed-loop system (7) is a time-varying stochastic differential equation. From the proof of Theorem 1, it can be seen that by constructing a stochastic Lyapunov function $V(t)$, the convergence of the closed-loop system is analyzed, where Assumption $\left(\mathbf{A}_{\mathbf{1}}\right)$ ensures the existence of a Lyapunov function, while Assumptions $\left(\mathbf{A}_{\mathbf{2}}\right)-\left(\mathbf{A}_{\mathbf{3}}\right)$ ensure that the mean square error $E\|\delta(t)\|^{2}$ converges to zero.

As stated in Theorem 1, Assumptions $\left(\mathbf{A}_{\mathbf{1}}\right)-\left(\mathbf{A}_{\mathbf{3}}\right)$ are sufficient conditions to guarantee that the protocol (3) is a strong mean square consensus protocol. In what follows, we will prove that when the measurement noises are zeros, Assumptions $\left(\mathbf{A}_{\mathbf{1}}\right)-\left(\mathbf{A}_{\mathbf{2}}\right)$ are necessary, too.

When $n_{j i}(t) \equiv 0$, the protocol (3) is reduced to

$$
u_{i}(t)=a(t)\left[\sum_{j \in N_{i}} a_{i j}\left(x_{j}(t)-x_{i}(t)\right)+b_{i}\left(x_{0}-x_{i}(t)\right)\right], t \geq 0, i=1,2, \cdots, N .
$$


In this case, applying the protocol (13) to the system (1), we obtain a tracking error equation as follows:

$$
d \delta(t)=a(t)\left(-L_{\overline{\mathcal{G}}}-B\right) \delta(t) d t .
$$

For this tracking error, we state the following result.

Theorem 2 For the system (1), if the protocol (3) is applied and $n_{j i}(t) \equiv 0, j \in N_{i}$, $i=1,2, \cdots, N$, then for any initial value $X(0), \lim _{t \rightarrow \infty}\|\delta(t)\|=0$ if and only if Assumptions $\left(\mathbf{A}_{\mathbf{1}}\right)-\left(\mathbf{A}_{\mathbf{2}}\right)$ hold.

Proof Sufficiency: Similar to the proof in Theorem 1, by using the unique positive definite solution $P$ of (9), we can define a Lyapunov function $V(t)=\delta^{\mathrm{T}}(t) P \delta(t)$, which together with (14) gives

$$
\frac{d V(t)}{d t}=-a(t) \delta^{\mathrm{T}}(t) \delta(t)
$$

Noticing that $P>0$, by (15) we have

$$
\frac{d V(t)}{d t} \leq-\frac{a(t)}{\lambda_{\max }(P)} V(t) .
$$

Integrating both sides of the above inequality results in

$$
V(t) \leq V(0) \exp \left\{-\frac{1}{\lambda_{\max }(P)} \int_{0}^{t} a(s) d s\right\} .
$$

This together with Assumption $\left(\mathbf{A}_{\mathbf{2}}\right)$ implies that

$$
\lim _{t \rightarrow \infty} V(t)=0 .
$$

Further, by $\|\delta(t)\|^{2} \leq \frac{V(t)}{\lambda_{\min }(P)}$, we have $\lim _{t \rightarrow \infty}\|\delta(t)\|=0$.

Necessity: By contradiction, suppose Assumption $\left(\mathbf{A}_{\mathbf{1}}\right)$ was not true. Then, there would exactly exist the following two cases ${ }^{[7]}$ :

(I) There exist at least two separate subgraphs in $\mathcal{G}$ without information exchange;

(II) There exist at least two vertices in $\mathcal{G}$ receiving no information.

For case $(\mathbf{I})$, suppose $\mathcal{V}_{1}$ and $\mathcal{V}_{2}$ are the sets of vertices of the two separate subgraphs, respectively. Then, $\mathcal{V}_{1} \cup \mathcal{V}_{2} \subseteq \mathcal{V}$, and for any $i \in \mathcal{V}_{1}$ and $j \in \mathcal{V}_{2}$, neither $(i, j)$ nor $(j, i)$ is in $\mathcal{E}$.

If the leader is not in $\mathcal{V}_{1} \cup \mathcal{V}_{2}$, then we can choose $x_{i}(0)=0, i \in \mathcal{V}_{1}$ and $x_{j}(0)=1, j \in \mathcal{V}_{2}$. In this case, by applying the protocol (13), there exist two Laplacian matrices $L_{1}$ and $L_{2}$ such that

$$
d X_{1}(t)=-a(t) L_{1} X_{1}(t) d t, \quad d X_{2}(t)=-a(t) L_{2} X_{2}(t) d t,
$$

where $X_{1}(t)$ and $X_{2}(t)$ are column vectors whose elements are composed of states of vertices in $\mathcal{V}_{1}$ and $\mathcal{V}_{2}$, respectively. Noting that $\mathbf{0}$ and $\mathbf{1}$ are the solutions of the above two equations, respectively, by the existence and uniqueness of solutions, we have $X_{1}(t) \equiv \mathbf{0}, X_{2}(t) \equiv \mathbf{1}$. Thus, $\lim _{t \rightarrow \infty} X_{1}(t) \neq \lim _{t \rightarrow \infty} X_{2}(t)$. This contradicts $\lim _{t \rightarrow \infty}\|\delta(t)\|=0$.

If the leader is in $\mathcal{V}_{1} \cup \mathcal{V}_{2}$, without loss of generality, suppose $0 \in \mathcal{V}_{1}$, then

$$
\begin{aligned}
& d X_{1}(t)=-a(t)\left(L_{1}+B_{1}\right) X_{1}(t) d t+a(t) B_{1}\left(\mathbf{1} \otimes x_{0}\right) d t, \\
& d X_{2}(t)=-a(t) L_{2} X_{2}(t) d t,
\end{aligned}
$$

where $B_{1}$ denotes the leader adjacency matrix associated with $\mathcal{V}_{1}$. Choosing $x_{i}(0)=x_{0}, i \in \mathcal{V}_{1}$; $x_{j}(0)=1+x_{0}, j \in \mathcal{V}_{2}$, and noting that $\mathbf{1} \otimes x_{0}$ and $\mathbf{1} \otimes\left(1+x_{0}\right)$ are solutions of the above two 
equations, respectively, by the existence and uniqueness of solutions, we get $X_{1}(t) \equiv \mathbf{1} \otimes x_{0}$ and $X_{2}(t) \equiv \mathbf{1} \otimes\left(1+x_{0}\right)$. Thus, $\lim _{t \rightarrow \infty} X_{1}(t) \neq \lim _{t \rightarrow \infty} X_{2}(t)$, which leads to a contradiction.

For case $(\mathbf{I I})$, there exist at least two vertices in $\mathcal{G}$ receiving no information, which implies that there exists at least one follower, say $i_{0}$, having no neighbors in $\mathcal{G}$. Selecting $x_{i_{0}}(0) \neq x_{0}$ and by applying (13) to the follower $i_{0}$, we have

$$
x_{i_{0}}(t)=x_{i_{0}}(0) \neq x_{0} .
$$

This contradicts $\lim _{t \rightarrow \infty}\|\delta(t)\|=0$.

From $(\mathbf{I})$ and $(\mathbf{I I})$, one can see that if $\lim _{t \rightarrow \infty}\|\delta(t)\|=0$, then $\mathcal{G}$ has a spanning tree. Namely, Assumption $\left(\mathbf{A}_{\mathbf{1}}\right)$ holds.

Now we only need to prove Assumption $\left(\mathbf{A}_{\mathbf{2}}\right)$.

By Assumption ( $\left.\mathbf{A}_{\mathbf{1}}\right)$, (15) holds. Thus,

$$
\frac{d V(t)}{d t} \geq-\frac{a(t)}{\lambda_{\min }(P)} V(t)
$$

Integrating both sides of the above inequality, we have

$$
V(t) \geq V(0) \exp \left\{-\frac{1}{\lambda_{\min }(P)} \int_{0}^{t} a(s) d s\right\} .
$$

Suppose Assumption $\left(\mathbf{A}_{\mathbf{2}}\right)$ was not true, namely, $\int_{0}^{t} a(s) d s<\infty$. Then, we would have

$$
\liminf _{t \rightarrow \infty} V(t) \geq V(0) \exp \left\{-\frac{1}{\lambda_{\min }(P)} \int_{0}^{\infty} a(s) d s\right\}>0, \forall \delta(0) \neq \mathbf{0} .
$$

By $\|\delta(t)\|^{2} \geq \frac{1}{\lambda_{\max }(P)} V(t)$, we get

$$
\liminf _{t \rightarrow \infty}\|\delta(t)\|^{2} \geq \frac{1}{\lambda_{\max }(P)} \liminf _{t \rightarrow \infty} V(t)>0, \forall \delta(0) \neq \mathbf{0} .
$$

This contradicts $\lim _{t \rightarrow \infty}\|\delta(t)\|=0$. Thus, Assumption $\left(\mathbf{A}_{2}\right)$ holds.

Remark 5 When $n_{j i}(t) \equiv 0$, from Theorem 2 it can be seen that Assumptions $\left(\mathbf{A}_{\mathbf{1}}\right)$ $\left(\mathbf{A}_{\mathbf{2}}\right)$ are necessary and sufficient conditions ensuring the followers can eventually follow the leader, where Assumption $\left(\mathbf{A}_{\mathbf{1}}\right)$ guarantees the connectivity of the communication topology which makes the state of each follower eventually equal the leader's; and Assumption $\left(\mathbf{A}_{\mathbf{2}}\right)$ guarantees the consensus error $\delta(t)$ converges to zero with a certain rate.

Remark 6 When $n_{j i}(t) \equiv 0$, in [6] and [12], the theory of stochastic matrices and nonnegative matrices was used for the convergence analysis. However, here Assumption $\left(\mathbf{A}_{\mathbf{2}}\right)$ cannot guarantee that there exists a positive constant $\alpha>0$ such that $a(t) \geq \alpha, \forall t \geq 0$ (for example, $a(t)=\frac{1}{t+1}, \forall t \geq 0$ ), and so, the positive entries of those off-diagonal ones in the state matrix of the closed-loop system (14) are not uniformly bounded away from zero. Thus, the main condition Assumption 1(b) in [12] does not hold, and the stochastic matrix methods used in [6] and [12] do not work here.

Remark 7 In Theorem $2, \mathcal{G}$ is not required to be balanced, which makes the tools of symmetric digraphs used in [10] do not work. Here, we propose a Lyapunov-based approach to deal with the problem.

As shown above, we analyze the convergence of the consensus protocol (3) under the fixed topologies. However, in most real communication processes, the communication links among 
the agents often change in time. For example, in the flocking and vehicle formation control, the communication topology depends on the environment of the flocking and the relative positions of the vehicles, which are usually changing in time. Thus, it will be interesting to study the convergence of the consensus protocol under switching topologies.

\subsection{Switching Topology}

Let $\sigma(t):[0, \infty) \longrightarrow \mathcal{I}_{\mathcal{T}^{*}}$ represent a switching signal that determines the communication topology. $\mathcal{T}^{*}$ is a set of digraphs with a common vertex set $\mathcal{V}$. Since at most a digraph with vertex set $\mathcal{V}$ has $N^{2}+N$ directed edges, the set $\mathcal{T}^{*}$ is finite and can be denoted as $\mathcal{T}^{*}=\left\{\mathcal{G}_{1}, \mathcal{G}_{2}, \cdots, \mathcal{G}_{N^{*}}\right\}$, where $N^{*}$ represents the total number of digraphs in $\mathcal{T}^{*}$ and $\mathcal{I}_{\mathcal{T}^{*}}=$ $\left\{1,2, \cdots, N^{*}\right\}$ is the index set associated with the elements of $\mathcal{T}^{*}$. We can rewrite the consensus protocol (3) as

$$
u_{i}(t)=a(t)\left[\sum_{j \in N_{i}\left(\mathcal{G}_{\sigma(t)}\right)} a_{i j}\left(\mathcal{G}_{\sigma(t)}\right)\left(y_{j i}(t)-x_{i}(t)\right)+b_{i}\left(\mathcal{G}_{\sigma(t)}\right)\left(y_{0 i}(t)-x_{i}(t)\right)\right], t \geq 0
$$

where $i=1,2, \cdots, N ; N_{i}\left(\mathcal{G}_{\sigma(t)}\right)$ is the set of neighbors of follower $i$ in the digraph $\mathcal{G}_{\sigma(t)}$; $a_{i j}\left(\mathcal{G}_{\sigma(t)}\right)(i, j=1,2, \cdots, N)$ is the element of the adjacency matrix of $\mathcal{G}_{\sigma(t)}$, and $B_{\mathcal{G}_{\sigma(t)}}=$ $\operatorname{diag}\left(b_{1}\left(\mathcal{G}_{\sigma(t)}\right), b_{2}\left(\mathcal{G}_{\sigma(t)}\right), \cdots, b_{N}\left(\mathcal{G}_{\sigma(t)}\right)\right)$ such that $b_{i}\left(\mathcal{G}_{\sigma(t)}\right)>0$ if and only if $0 \in N_{i}\left(\mathcal{G}_{\sigma(t)}\right)$.

Let $\delta(t)=X(t)-\mathbf{1} \otimes x_{0}$ as in Subsection 5.1. Then, by substituting the protocol (16) to the system $(1)$, we get

$$
d \delta(t)=a(t)\left(-L_{\overline{\mathcal{G}}_{\sigma(t)}}-B_{\mathcal{G}_{\sigma(t)}}\right) \delta(t) d t+a(t) G_{\mathcal{G}_{\sigma(t)}} d W(t),
$$

where $L_{\overline{\mathcal{G}}_{\sigma(t)}}$ is the Laplacian matrix of the digraph $\overline{\mathcal{G}}_{\sigma(t)}$ formed by $N$ followers in the digraph $\mathcal{G}_{\sigma(t)}$ and

$$
G_{\mathcal{G}_{\sigma(t)}}=\operatorname{diag}\left(\sqrt{b_{1}^{2}\left(\mathcal{G}_{\sigma(t)}\right)+\sum_{j \in N_{1}\left(\mathcal{G}_{\sigma(t)}\right)} a_{1 j}^{2}\left(\mathcal{G}_{\sigma(t)}\right)}, \cdots, \sqrt{b_{N}^{2}\left(\mathcal{G}_{\sigma(t)}\right)+\sum_{j \in N_{N}\left(\mathcal{G}_{\sigma(t)}\right)} a_{N j}^{2}\left(\mathcal{G}_{\sigma(t)}\right)}\right) .
$$

In the sequel, we will analyze the convergence of the consensus protocol (16) based on the closed-loop system (17). Denote $M_{\sigma(t)}=L_{\overline{\mathcal{G}}_{\sigma(t)}}+B_{\mathcal{G}_{\sigma(t)}}$, which will play an important role in the subsequent convergence analysis. First, we introduce a property of $M_{\sigma(t)}$.

Proposition 1 Suppose the digraph $\overline{\mathcal{G}}_{\sigma(t)}$ is balanced. Then, $M_{\sigma(t)}+M_{\sigma(t)}^{\mathrm{T}}$ is positive definite if and only if the digraph $\mathcal{G}_{\sigma(t)}$ has a spanning tree.

Proof Sufficiency: Since the subgraph $\overline{\mathcal{G}}_{\sigma(t)}=\left(\overline{\mathcal{V}}, \overline{\mathcal{E}}_{\mathcal{G}_{\sigma(t)}}, \overline{\mathcal{A}}_{\mathcal{G}_{\sigma(t)}}\right)$ of $\mathcal{G}_{\sigma(t)}$ is balanced, by Lemma $1, \frac{L_{\overline{\mathcal{G}}_{\sigma(t)}}+L_{\overline{\mathcal{G}}_{\sigma(t)}^{\mathrm{T}}}}{\frac{2}{\mathcal{G}}}$ is the Laplacian matrix of $\widehat{\overline{\mathcal{G}}}_{\sigma(t)}=\left(\overline{\mathcal{V}}, \widehat{\overline{\mathcal{E}}}_{\mathcal{G}_{\sigma(t)}}, \widehat{\overline{\mathcal{A}}}_{\mathcal{G}_{\sigma(t)}}\right)$, which is the mirror digraph of $\overline{\mathcal{G}}_{\sigma(t)}$. Thus,

$$
\widehat{L}_{\sigma(t)}=\left(\begin{array}{cc}
0 & 0 \\
-B_{\mathcal{G}_{\sigma(t)}} \cdot \mathbf{1} & \frac{M_{\sigma(t)}+M_{\sigma(t)}^{\mathrm{T}}}{2}
\end{array}\right)
$$

is the Laplacian matrix of the digraph $\widehat{\mathcal{G}}_{\sigma(t)}=\left(\mathcal{V}, \widehat{\mathcal{E}}_{\sigma(t)}, \widehat{\mathcal{A}}_{\sigma(t)}\right)$, where

$$
\widehat{\mathcal{A}}_{\sigma(t)}=\left(\begin{array}{cc}
0 & 0 \\
B_{\mathcal{G}_{\sigma(t)}} \cdot \mathbf{1} & \widehat{\overline{\mathcal{A}}}_{\mathcal{G}_{\sigma(t)}}
\end{array}\right) \text {. }
$$


By Lemma 2, $\widehat{L}_{\sigma(t)}$ has at least one zero eigenvalue and all of the nonzero eigenvalues are in the open right half plane. Since $\mathcal{G}_{\sigma(t)}$ has a spanning tree and by the definition of the mirror digraph, $\widehat{\mathcal{G}}_{\sigma(t)}$ must have a spanning tree. Thus, $\widehat{L}_{\sigma(t)}$ has exactly one zero eigenvalue, and hence, all the eigenvalues of $M_{\sigma(t)}+M_{\sigma(t)}^{\mathrm{T}}$ are in the open right half plane. Further, by the symmetry of $M_{\sigma(t)}+M_{\sigma(t)}^{\mathrm{T}}, M_{\sigma(t)}+M_{\sigma(t)}^{\mathrm{T}}$ is positive definite.

Necessity: Since

$$
L_{\mathcal{G}_{\sigma(t)}}=\left(\begin{array}{cc}
0 & 0 \\
-B_{\mathcal{G}_{\sigma(t)}} \cdot \mathbf{1} & M_{\sigma(t)}
\end{array}\right)
$$

is the Laplacian matrix of $\mathcal{G}_{\sigma(t)}$, by Lemma $2, M_{\sigma(t)}$ has either at least one zero eigenvalue or eigenvalues with positive real-parts. If $M_{\sigma(t)}$ has zero eigenvalue and $\alpha$ is the corresponding eigenvector, then $\alpha \neq 0$ and $M_{\sigma(t)} \alpha=0$. A straightforward calculation shows that $\alpha^{\mathrm{T}}\left(M_{\sigma(t)}+M_{\sigma(t)}^{\mathrm{T}}\right) \alpha=0$, which in turn implies that $M_{\sigma(t)}+M_{\sigma(t)}^{\mathrm{T}}$ is not positive definite. This is a contradiction. Thus, $M_{\sigma(t)}$ has no zero eigenvalues, and hence, $L_{\mathcal{G}_{\sigma(t)}}$ has exactly one zero eigenvalue. This together with Lemma 2 implies that $\mathcal{G}_{\sigma(t)}$ has a spanning tree.

Based on Proposition 1 and the fact that $\mathcal{T}^{*}$ is finite,

$$
\begin{aligned}
& \lambda^{*}=\lambda_{\max }\left\{M_{\sigma(t)}+M_{\sigma(t)}^{\mathrm{T}} \mid \mathcal{G}_{\sigma(t)} \text { has a spanning tree }\right\}>0, \\
& \mu^{*}=\lambda_{\min }\left\{M_{\sigma(t)}+M_{\sigma(t)}^{\mathrm{T}} \mid \mathcal{G}_{\sigma(t)} \text { has a spanning tree }\right\}>0
\end{aligned}
$$

are well defined.

The main result of this section can be summarized as follows:

Theorem 3 For the system (1) with the protocol (16), if for any $t \geq 0, \overline{\mathcal{G}}_{\sigma(t)}$ is balanced, and $\mathcal{G}_{\sigma(t)}$ has a spanning tree, then under Assumptions $\left(\mathbf{A}_{\mathbf{2}}\right)-\left(\mathbf{A}_{\mathbf{3}}\right)$,

$$
\lim _{t \rightarrow \infty} E\|\delta(t)\|^{2}=0 .
$$

Proof By (17) and the Itô formula, we get

$$
d\|\delta(t)\|^{2} \leq-\mu^{*} a(t)\|\delta(t)\|^{2} d t+a^{2}(t) \operatorname{tr}\left(G_{\mathcal{G}_{\sigma(t)}} G_{\mathcal{G}_{\sigma(t)}}^{\mathrm{T}}\right) d t+2 a(t) \delta^{\mathrm{T}}(t) G_{\mathcal{G}_{\sigma(t)}} d W(t) .
$$

Noticing $\sigma(t) \in \mathcal{I}_{\mathcal{T}^{*}}=\left\{1,2, \cdots, N^{*}\right\}$ and the fact that $\mathcal{I}_{\mathcal{T}^{*}}$ is finite, we can define

$$
\beta_{0}=\max \left\{\operatorname{tr}\left(G_{\mathcal{G}_{\sigma(t)}} G_{\mathcal{G}_{\sigma(t)}}^{\mathrm{T}}\right) \mid \forall \sigma(t) \in \mathcal{I}_{\mathcal{T}^{*}}\right\}>0 .
$$

Thus, by (18) we have

$$
d\|\delta(t)\|^{2} \leq-\mu^{*} a(t)\|\delta(t)\|^{2} d t+\beta_{0} a^{2}(t) d t+2 a(t) \delta^{\mathrm{T}}(t) G_{\mathcal{G}_{\sigma(t)}} d W(t) .
$$

Similar to the proof of (11) in Theorem 1, one can get

$$
E \int_{t_{0}}^{t} a(s) \delta^{\mathrm{T}}(s) G_{\mathcal{G}_{\sigma(s)}} d W(s)=0, \quad \forall 0 \leq t_{0} \leq t
$$

This together with (19) implies that for any $t \geq 0$ and $h>0$,

$$
E\left[\|\delta(t+h)\|^{2}\right]-E\left[\|\delta(t)\|^{2}\right] \leq-\mu^{*} \int_{t}^{t+h} a(s) E\left[\|\delta(s)\|^{2}\right] d s+\beta_{0} \int_{t}^{t+h} a^{2}(s) d s,
$$


or equivalently,

$$
\frac{E\left[\|\delta(t+h)\|^{2}\right]-E\left[\|\delta(t)\|^{2}\right]}{h} \leq \frac{-\mu^{*} \int_{t}^{t+h} a(s) E\left[\|\delta(s)\|^{2}\right] d s+\beta_{0} \int_{t}^{t+h} a^{2}(s) d s}{h} .
$$

Further,

$$
\begin{aligned}
\limsup _{h \rightarrow 0^{+}} \frac{E\left[\|\delta(t+h)\|^{2}\right]-E\left[\|\delta(t)\|^{2}\right]}{h} & \leq \limsup _{h \rightarrow 0^{+}} \frac{-\mu^{*} \int_{t}^{t+h} a(s) E\left[\|\delta(s)\|^{2}\right] d s+\beta_{0} \int_{t}^{t+h} a^{2}(s) d s}{h} \\
& =-\mu^{*} a(t) E\left[\|\delta(t)\|^{2}\right]+\beta_{0} a^{2}(t) .
\end{aligned}
$$

Thus, by the comparison principle ${ }^{[16]}$, we have

$$
E\left[\|\delta(t)\|^{2}\right] \leq E\left[\|\delta(0)\|^{2}\right] \exp \left\{-\mu^{*} \int_{0}^{t} a(s) d s\right\}+\beta_{0} \int_{0}^{t} a^{2}(s) \exp \left\{-\mu^{*} \int_{s}^{t} a(\tau) d \tau\right\} d s .
$$

The remaining of the proof is largely similar to that of Theorem 1 , and hence, omitted here.

Remark 8 From Theorem 3, it can be seen that even under the switching topologies, the consensus protocol (3) can still guarantee the state of each follower converges to that of the leader in mean square, i.e., (3) is a strong mean square consensus protocol.

\section{Numerical Example}

In this section, we will use an example to demonstrate the leader-following consensus after applying the consensus protocol (3) to each follower.

Example 1 We consider a system consisting of one leader indexed by 0 and two followers indexed by 1 and 2, respectively. $x_{0}=1$ is the state of the leader and the dynamics of the $i$ th follower is described as follows:

$$
\dot{x}_{i}(t)=u_{i}(t), \quad i=1,2 .
$$

Assume the initial states of the two followers indexed by 1 and 2 are $x_{1}(0)=2$ and $x_{2}(0)=-2$, respectively.

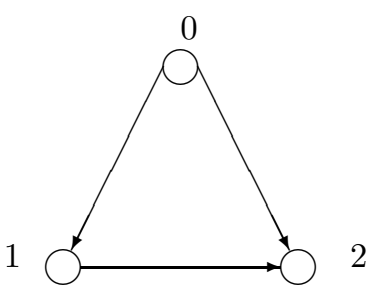

Figure 1 The communication topology among agents

The communication topology within the above leader-following MAS is described by the digraph $\mathcal{G}^{*}=\left(\mathcal{V}^{*}, \mathcal{E}^{*}, \mathcal{A}^{*}\right)$ as shown in Figure 1, where

$$
\mathcal{V}^{*}=\{0,1,2\}, \quad \mathcal{E}^{*}=\{(0,1),(0,2),(1,2)\}, \quad \mathcal{A}^{*}=\left[\begin{array}{lll}
0 & 0 & 0 \\
1 & 0 & 0 \\
1 & 1 & 0
\end{array}\right] .
$$

In this example, $\left\{n_{j i}(t), i, j=0,1,2\right\}$ are independent standard white noises. 


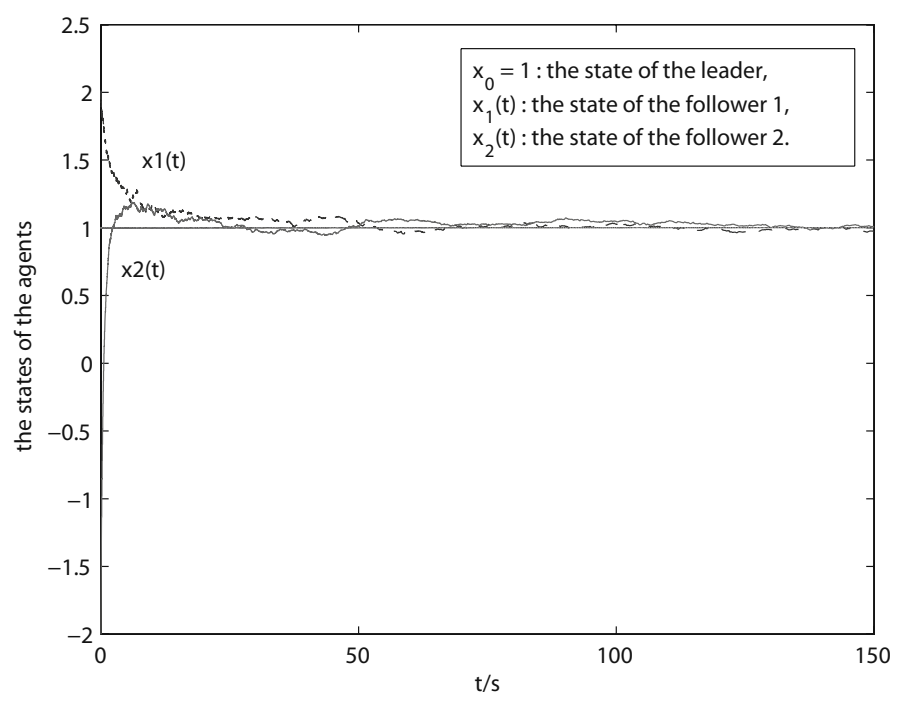

Figure 2 States of the followers and the leader

To make the state of each follower converge to that of the leader, we apply the consensus protocol (3) to the system (21) and choose the consensus gain function $a(t)=\frac{1}{t+1}, t \geq 0$. The simulation result for the follower following the leader is shown in Figure 2.

From Figure 2, one can see that after applying the consensus protocol (3), the states of follower 1 and 2 will eventually converge to that of the leader as time goes on.

\section{Conclusions}

This paper studies the consensus control for leader-following MASs under measurement noises. For the leader-following MAS, a measurement-based distributed protocol is designed and convergence properties of the protocol are analyzed by using the tools of stochastic analysis and algebraic graph theory. Under fixed topologies, the protocol is proved to ensure the state of each follower converge to that of the leader in mean square. Also, under the switching topology case, the result still holds if the subgraph formed by followers is balanced.

It is worth pointing out that this paper is only a preliminary step on leader-following consensus under measurement noises. When the state of the leader is not a constant, as Ren ${ }^{[17]}$ pointed out, the extension of consensus algorithms from a constant reference to a time-varying one is non-trivial, it will be challenging to design consensus protocols to guarantee the convergence of the state of each follower to that of the time-varying leader under measurement noises. Of course, There are many other topics worth investigating, such as, how to design protocols for time-delay cases, and how to get almost sure consensus protocols, etc.

\section{References}

[1] R. Olfati-Saber, Flocking for multi-agent dynamic systems: Algorithms and theory, IEEE Transactions on Automatic Control, 2006, 51(3): 401-420.

[2] N. E. Leonard and E. Fiorelli, Virtual leaders, artifical potentials and coordinated control of groups, 
in Proceedings of the 40th IEEE Conference on Decision and Control, Orland, Florida, 2001.

[3] C. Belta and V. Kumar, Trajectory design for formations of robots by kinetic energy shaping, in Proceedings of the 2002 IEEE International Conference on Robotics and Automation, Washington, DC, USA, 2002.

[4] W. Ren, R. W. Beard and T. W. Mclain, Coordination variables and consensus building in multiple vehicle systems, in Cooperative Control: A Post-Workshop Volume 2003 Block Island Workshop on Cooperative Control (ed. by V. Kumar, N. Leonard, and A. S. Morse), Springer-Verlag Series: Lecture Notes in Control and Information Sciences, Springer, Berlin, 2004, 171-188.

[5] C. Cutts and J. Speakman, Energy savings in formation flight of pink-footed geese, Journal of Experimental Biology, 1994, 189(1): 251-261.

[6] A. Jadbabaie, J. Lin, and A. S. Morse, Coordination of groups of mobile autonomous agents using nearest neighbor rules, IEEE Transactions on Automatic Control, 2003, 48(6): 988-1001.

[7] W. Ren and R. W. Beard, Consensus seeking in multiagent systems under dynamically changing interaction topologies, IEEE Transactions on Automatic Control, 2005, 50(5): 655-661.

[8] Y. Hong, J. Hu, and L. Gao, Tracking control for multi-agent consensus with an active leader and variable topology, Automatica, 2005, 42(7): 1177-1182.

[9] W. Ren, R. W. Beard, and E. M. Atkins, A survey of consensus problems in multi-agent coordination, in Proceedings of the 2005 American Control Conference, Portland, OR, 2005.

[10] T. Li and J. F. Zhang, Mean square average consensus under measurement noises and fixed topologies: Necessary and sufficient conditions, Automatica, 2009, 45(8): 1929-1936.

[11] T. Li and J. F. Zhang, Consensus conditions of multi-agent systems with time-varying topologies and stochastic communication noises, provisionally accepted by IEEE Transactions on Automatic Control, 2010, 55(9).

[12] J. N. Tsitsiklis, D. P. Bertsekas, and M. Athans, Distributed asynchronous deterministic and stochastic gradient optimization algorithms, IEEE Transactions on Automatic Control, 1986, 31(9): 803-812.

[13] R. Olfati-Saber and R. M. Murray, Consensus problems in networks of agents with switching topology and time-delays, IEEE Transactions on Automatic Control, 2004, 49(9): 1520-1533.

[14] M. Huang and J. H. Manton, Coordination and consensus of networked agents with noisy measurement: stochastic algorithms and asymptotic behavior, SIAM Journal on Control and Optimization, 2009, 48(1): 134-161.

[15] M. B. Nevel'son and R. Z. Has'minskii, Stochastic Approximation and Recursive Estimation, American Mathematical Society, Providence, 1976.

[16] A. N. Michel and R. K. Miller, Qualitative Analysis of Large Scale Dynamical Systems, Academic Press, New York, 1977.

[17] W. Ren, Multi-vehicle consensus with a time-varying reference state, Systems and Control Letters, $2007, \mathbf{5 6}(7-8)$ : 474-483. 